\title{
Studies on Auditory Thresholds in Normal Man and in Patients with Adrenal Cortical Insufficiency: The Role of Adrenal Cortical Steroids*
}

\author{
Robert I. Henkin, $†$ Robert E. McGlone, Robert Daly, and \\ Frederic C. BartTer \\ (From the Clinical Endocrinology Branch, National Heart Institute, and the National Institute \\ of Dental Research, Bethesda, Md.; and Gallaudet College, Washington, D. C.)
}

Summary. Auditory thresholds for sinusoidal tones were determined in eight patients with adrenal cortical insufficiency (four with Addison's disease and four with panhypopituitarism) and compared to those in normal volunteers. In adrenal cortical insufficiency (ACI) the auditory detection sensitivity is significantly more acute than that of normal subjects over most of the frequency range, but especially in the region of greatest hearing sensitivity of normal subjects, 1,000 to 2,000 cycles per second (cps).

Treatment of the patients with deoxycorticosterone acetate decreased serum potassium concentration and produced gains in body weight but did not alter auditory detection thresholds.

Treatment with prednisolone or with maintenance doses of carbohydrateactive steroids returned the auditory detection threshold to normal in every patient tested.

The mechanism by which carbohydrate-active steroids affect the sensitivity of the nervous system to sound is not known. However, since the senses of taste, smell, and hearing are all affected in similar fashion by their removal and replacement, there appears to be a generalized increase in sensitivity to all sensory stimuli in patients with ACI not receiving steroids. These hormones may play a significant role in maintaining the level of responsiveness of the sensory system to incoming stimuli.

\section{Introduction}

It has been shown previously that patients with untreated adrenal cortical insufficiency exhibit markedly increased detection sensitivity for all four modalities of taste and for the smells of various vapors $(1,2)$. Treatment of the patients with ACI with deoxycorticosterane acetate (DOCA) for 2 to 9 days produced no alteration in taste or smell thresholds, but treatment with carbohydrate-active steroids for 18 to 36 hours returned taste and smell thresholds to normal $(1,2)$. These studies indicated further that

* Submitted for publication May 26, 1966; accepted November 28, 1966.

$\dagger$ Address requests for reprints to Dr. Robert I. Henkin, Clinical Endocrinology Branch, National Heart Institute, Bethesda, Md. 20014. changes in extracellular sodium or potassium concentration or in extracellular fluid volume could not account for these effects $(1,2)$.

The present study was designed to evaluate the detection thresholds of patients with ACI with respect to another sensory modality, audition, and to explore the effects of treatment with adrenal cortical hormones and adrenocorticotropin on auditory thresholds.

\section{Methods}

The subjects of this study were 15 normal volunteers aged 18 to 26 (mean age, 21.6); 10 normal volunteers aged 39 to 61 (mean age, 47.1) ; 4 patients with anterior pituitary insufficiency aged 14 to 57 ; and 4 patients with adrenal cortical insufficiency aged 19 to 52 . All patients with adrenal cortical insufficiency had clinical features of this disease, urinary 17-hydroxycorticosteroids that were 


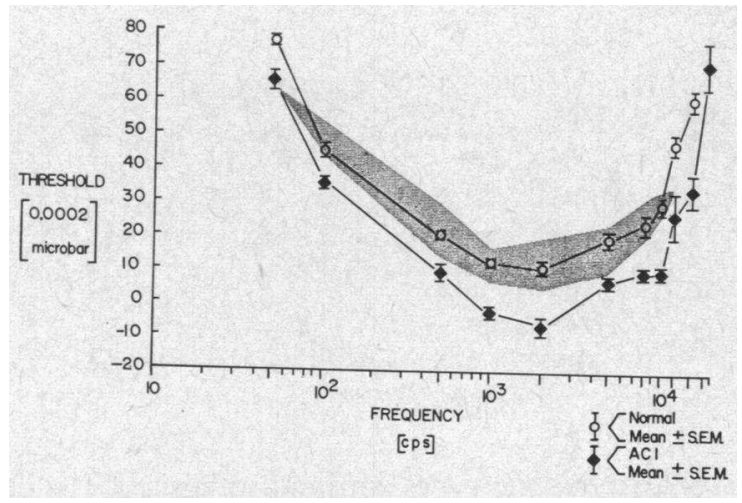

Fig. 1. COMPARISON OF DETECTION THRESHOLDS FOR SINUSOIDAL TONES IN 15 NORMAL VOLUNTEERS, MEAN AGE 21.6, WITH THOSE OBTAINED IN 4 PATIENTS WITH ADRENAL CORTICAL INSUFFICIENCY (ACI), MEAN AGE 19.9. The open circles represent the mean threshold responses of the normal volunteers, the black rectangles the mean threshold responses of the patients with ACI. The lines extending above and below the symbols represent 1 SEM. The upper limits of the crosshatched area represent the threshold values obtained by VonBekesy (7) and Steinberg and associates (8). The lower limits represent those obtained by Sivian and White (6). The data for the normal volunteers fall approximately midway between these limits. At each frequency, mean threshold for the patients with ACI was significantly below that of the normal volunteers. cps $=$ cycles per second.

below $2 \mathrm{mg}$ per 24 hours and did not increase with ACTH, $40 \mathrm{U}$ given intravenously over 8 hours each day for 4 days (except for V.M., whose urinary 17-hydroxycorticosteroids rose to $5.0 \mathrm{mg}$ per 24 hours on the fourth day of ACTH). All patients with anterior pituitary insufficiency had hypothyroidism, hypogonadism, and adrenal cortical insufficiency, with urinary 17-hydroxycorticosteroids below $2.2 \mathrm{mg}$ per 24 hours, rising above $10 \mathrm{mg}$ per 24 hours after infusion of $\mathrm{ACTH}, 40 \mathrm{U}$ given intravenously over 8 hours each day for 2 or more days. Taste and smell thresholds have been reported previously for four of these eight patients $(1,2)$. All patients remained on an air-conditioned metabolic ward and ate a regular diet, which was well tolerated even when they were not receiving treatment. Sodium intake was 100 to $200 \mathrm{mEq}$ per day. Body weight, determined with metabolic scales daily on arising, was used to provide a gross estimate of changes in the volume of body fluids. None of the subjects or patients gave a history of gross hearing loss or acute or chronic ear disease. Otolaryngological examination revealed no gross abnormalities in any of the normal subjects or patients with ACI. The patients were studied under the following five conditions: a) untreated for 4 or more days; $b$ ) treated with DOCA, $20 \mathrm{mg}$ per day, for 1 to 9 days; $c$ ) treated with prednisolone, $20 \mathrm{mg}$ per day, for 2 to 7 days; $d$ ) treated with ACTH, $40 \mathrm{U}$ intravenously over 8 hours, for 4 days; and $e)$ treated with maintenance dosages of $9 \alpha$-fluorohydro- cortisone ( 0.05 to $0.10 \mathrm{mg}$ per day) and of prednisolone ( 5.0 to $7.5 \mathrm{mg}$ per day) or dexamethasone $(0.5 \mathrm{mg}$ per day).

Auditory thresholds were evaluated by measuring each subject's minimal sensitivity for a sinusoidal stimulus over a wide range of frequencies. Thresholds were measured independently by two experimenters on separate occasions during the afternoon hours. Two of the experimenters (R.M., R.D.) participated in the study on a double blind basis. The threshold measurements thus determined by any two of three observers in both patients and normal volunteers differed by less than 5 decibels $(\mathrm{db})$ within any given subject over the entire range of frequencies tested for any given treatment condition.

Threshold determinations were made with the subjects and patients comfortably seated alone in an arm chair in an Industrial Acoustic 1204 sound chamber. Acoustic signals, provided by a Southwestern Industrial Electronics model M-2 R-C oscillator, were presented to the subjects over short intervals of time. The signal was attenuated by a Hewlett Packard model 350 B attenuator and then presented to the left and right ear of each subject separately through a Knight KN 848 circumaural earphone.

We obtained thresholds monoaurally in both ears of each patient utilizing the method of limits. Order of presentation to left or right ear was randomized to eliminate order and practice effects. The data reported here are the mean thresholds for the second or better ear tested. Threshold values for normal subjects obtained by these techniques are directly comparable to, and in good agreement with, those reported by other investigators [(3-8), Figure 1].

TABLE I

Auditory detection thresholds in normal subjects

\begin{tabular}{cll}
\hline Frequency & $\begin{array}{c}\text { Detection } \\
\text { threshold* }\end{array}$ & \multicolumn{1}{c}{$\begin{array}{c}\text { Detection } \\
\text { threshold* }\end{array}$} \\
\hline$c p s \dagger$ & & \\
50 & $77.4 \pm 1.9$ & $77.1 \pm 0.7$ \\
70 & $61.8 \pm 5.8$ & $70.1 \pm 1.6$ \\
100 & $45.3 \pm 2.2$ & $60.0 \pm 1.9$ \\
200 & $29.1 \pm 1.2$ & $50.0 \pm 1.0$ \\
500 & $20.8 \pm 1.2$ & $31.6 \pm 1.8$ \\
1,000 & $12.7 \pm 1.2$ & $23.1 \pm 2.1$ \\
2,000 & $11.3 \pm 2.1$ & $22.6 \pm 4.0$ \\
5,000 & $20.0 \pm 2.2$ & $33.8 \pm 4.4$ \\
8,000 & $24.5 \pm 3.0$ & $40.3 \pm 3.7$ \\
10,000 & $30.0 \pm 2.7$ & $54.0 \pm 4.1$ \\
12,000 & $48.1 \pm 3.2$ & \\
15,000 & $61.4 \pm 2.9$ & \\
Mean range of response (cps) & $50.5-15,500$ & $37.5-11,625$ \\
Mean age of volunteers & 21.6 & 47.1 \\
(years) & & \\
Age range (years) & $18-26$ & $39-61$ \\
Number of volunteers & 15 & 10 \\
\hline
\end{tabular}

* Re 0.0002 microbar; values given are means \pm standard errors.

t Cycles per second. 
The eight patients with ACI were found to have a bimodal age distribution: four had a mean age of 19.9 years, whereas the other four had a mean age of 48.0 years. To compare data from the patients with those from the normal subjects, it was necessary to select two groups of normal subjects with ages comparable to those of the patients. Thus, 15 younger normal volunteers, ranging in age from 18 to 26 years, and 10 older ones, ranging in age from 39 to 61 years, were chosen. In order to make meaningful comparisons between the patients and the normal volunteers without introducing the effect of age upon hearing thresholds as an uncontrolled variable, we have treated threshold data for the younger and older subjects separately in this paper.

\section{Results}

Auditory thresholds in normal subjects. The mean detection thresholds for each frequency tested in the 15 younger and the 10 older normal volunteers are presented in Table I. The means demonstrate that the greatest sensitivity of normal hearing is between 1,000 and 2,000 cps in both groups. For the younger subjects, the smallest variation occurs at those frequencies where sensitivity tends to be the greatest. For the older subjects, variance is increased at frequencies of 2,000 cps and above. This greater variation at higher frequencies in the older subjects is presumably a manifestation of the aging effects on the auditory thresholds. The means determined for the younger subjects are similar to those determined by other investigators, falling between the threshold curves reported by VonBekesy (7) and Steinberg, Montgomery, and Gardner (8) and those reported by Sivian and White (6) (Figure 1). The mean frequency range of responses for these subjects was 50.5 to $15,500 \mathrm{cps}$. The means for the older subjects are similar to those for the younger subjects for frequencies of $70 \mathrm{cps}$ or below, but significantly higher for all frequencies of $100 \mathrm{cps}$ or above. The pattern of responses is similar to that described by other investigators (9-11). The mean range of frequency responses for these older subjects was 37.5 to 11,625 cps. Thus, not only is there a marked decrease in frequency response in the older, as compared to the younger, subjects at the high end of the frequency spectrum, but there is also a slight increase in the frequency response at the lower end of the spectrum.

Auditory thresholds in patients with ACI receiving no steroid. The detection threshold for each frequency in each patient with untreated adrenal cortical insufficiency is presented in Table II. The younger patients consist of two with Addison's disease and two with panhypopituitarism (R.E., P.K., E.N., and L.D.). The older patients also consist of two with Addison's disease and two with panhypopituitarism (R.P., V.M., H.L., and E.B.). The detection thresholds for the patients are significantly lower than those for the normal subjects of comparable ages at all frequencies tested between 50 and 10,000 cps. The greatest increase in sensitivity was found at about 1,000 to $2,000 \mathrm{cps}$, the frequency range in which maximal hearing sensitivity is found in normal subjects. At these frequencies, the patients could detect tones that were 11 to $24 \mathrm{db}$ below those detected by comparable normal subjects. In no instance did the range of thresholds observed for the frequencies between 100 and 5,000 cps overlap the range for comparable normal subjects. Thresholds significantly lower than normal were found at both 12,000 and $15,000 \mathrm{cps}$ for the younger patients. However, at the higher frequencies, differences between the older patients and the older subjects became smaller. Mean detection thresholds for the older patients are significantly lower than those of the younger normal subjects at 500, 1,000, and 2,000 cps. This emphasizes the extreme sensitivity of the auditory system in patients with untreated ACI ; the older patients are, on the average, 28 years older than the younger normal subjects.

The general pattern of hearing responses is the same in both patients and normal subjects (Figure 1 ), but the frequency range for both patient groups is markedly expanded, as compared to that of comparable normal subjects, particularly at the high end of the frequency spectrum. The mean frequency range for the younger patients, off treatment, is an average of $21 \mathrm{cps}$ lower at the low end of the frequency spectrum and an average of 3,375 cps higher at the high end, as compared to the range for the younger normal subjects. For the older patients, the upper frequency range has been increased by an average of $1,775 \mathrm{cps}$, as compared to that for the older normal subjects.

Auditory thresholds in patients with ACI treated with DOCA. The detection threshold for each frequency in each patient with ACI treated with DOCA alone is presented in Table III. The means of the detection thresholds for both groups of patients are virtually the same as those observed 


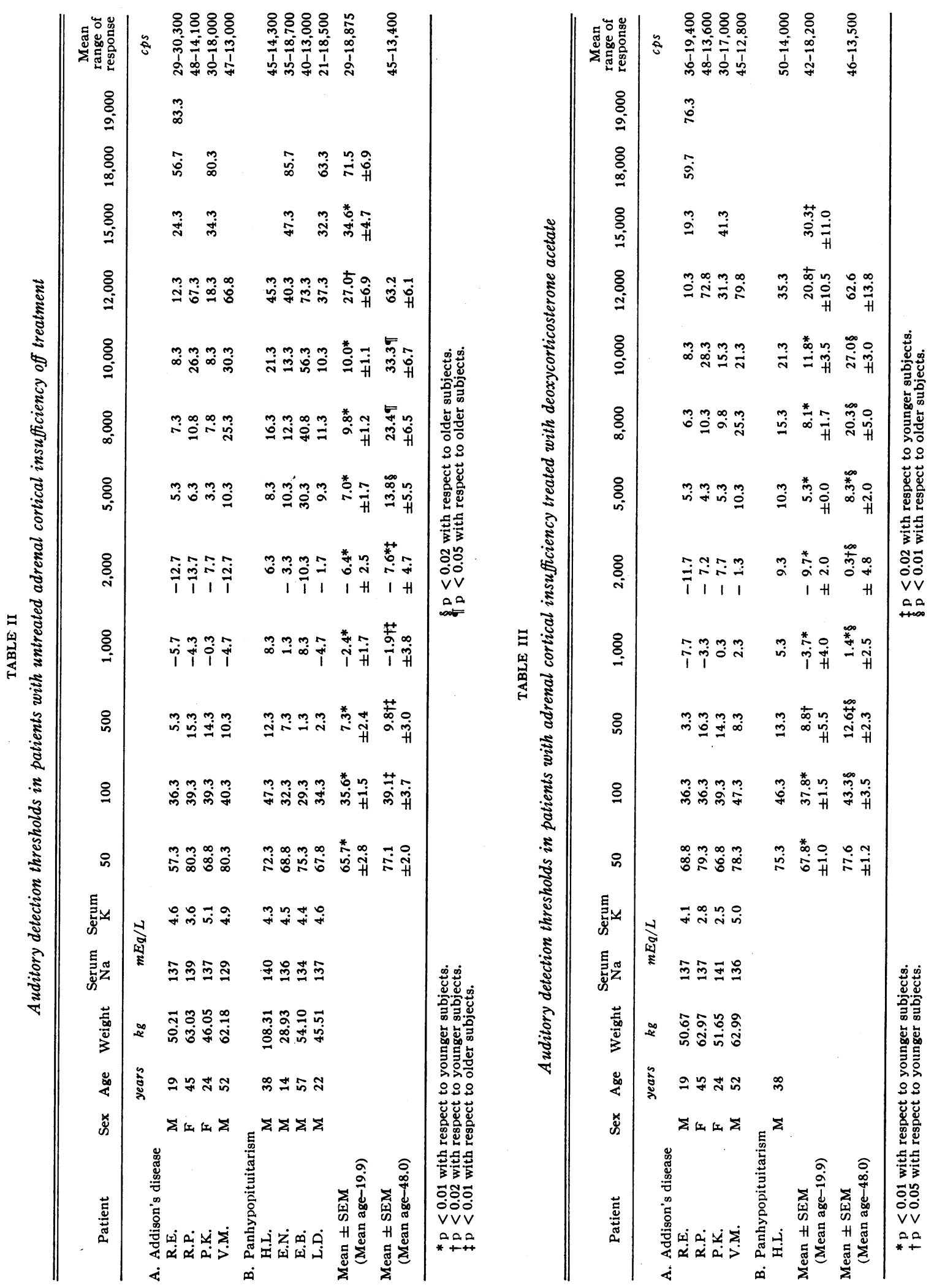


off treatment (Table II), despite decreases of serum potassium concentration and gains in body weight.

Auditory thresholds in patients with ACI treated with prednisolone. The detection threshold for each frequency in each patient with ACI treated only with prednisolone, $20 \mathrm{mg}$ per day, for 2 to 4 days, is presented in Table IV. In all patients, the mean detection thresholds returned to normal levels in the lower and middle frequency ranges after treatment for 2 to 4 days. ${ }^{1}$ The average outer limits of the frequency range for the younger patients decreased to 47 to $16,750 \mathrm{cps}$, which are essentially the same as the limits observed in the younger normal subjects. A similar decrease was observed in the older patients. This return of threshold to normal occurred also after treatment with maintenance doses of carbohydrate-active steroid alone or with maintenance doses of sodium-potassium-active steroid. Similarly, in those patients with panhypopituitarism and in the patient with ACI with some remaining adrenal cortical function, treatment with ACTH over 4 days returned hearing thresholds to normal in the same manner as that observed after treatment with carbohydrate-active steroids ( $\mathrm{Ta}$ ble IV).

\section{Discussion}

Determination of detection thresholds for a sinusoidal tone over the entire frequency range of hearing has shown that thresholds for all frequencies tested were lowered in untreated patients with adrenal cortical insufficiency as compared to normal subjects. This increase in sensitivity extends over the entire frequency range but is greatest between 1,000 and 2,000 cps, the region of greatest hearing sensitivity in normal subjects. The frequency response pattern of these patients is essentially the same as that of normal subjects (Figure 1).

Treatment of these patients with DOCA alone for as long as 9 days did not alter the abnormal hearing thresholds in any discernible manner. This observation is similar to those made previously, in which the increased sensitivity for taste

1 In some patients, treatment with carbohydrate-active steroids for periods longer than 4 days was required before detection thresholds returned to normal in the higher frequency range (above $10,000 \mathrm{cps}$ ).

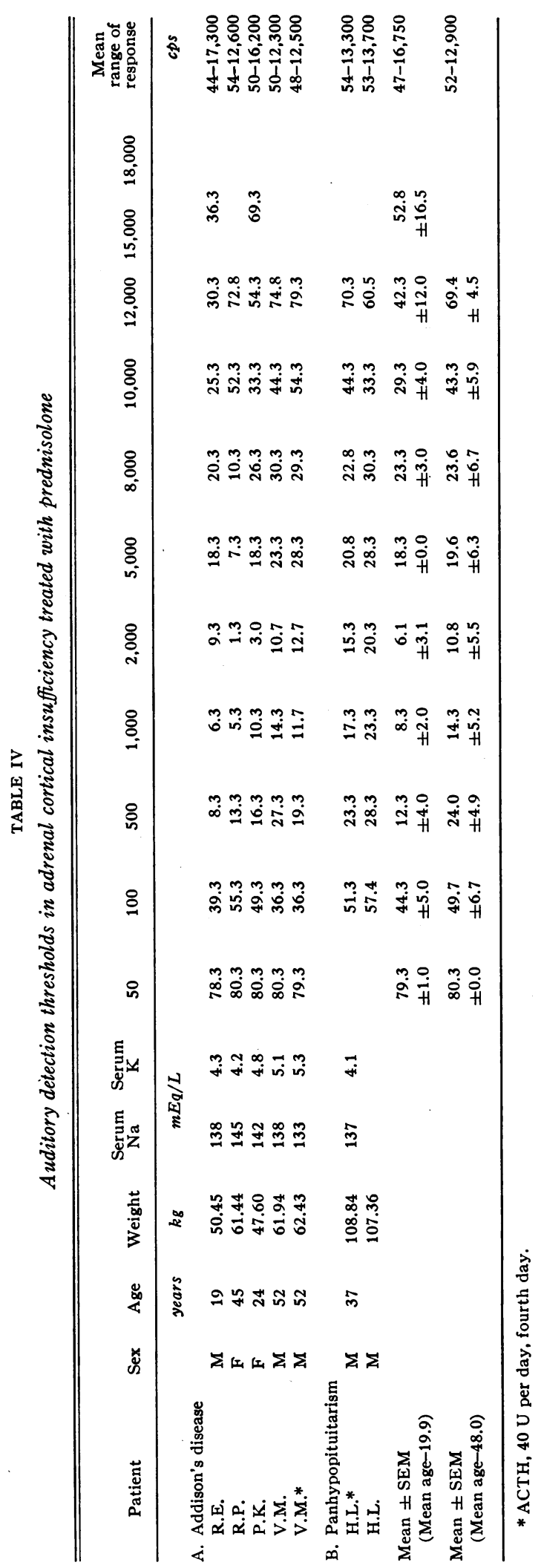


and olfaction observed in these patients did not change after treatment with DOCA $(1,2)$.

Treatment of the patients with ACI with carbohydrate-active steroid returned hearing thresholds to normal, as previously shown for the senses of taste and smell. Although taste detection returned to normal 18 to 36 hours after treatment with large doses of prednisolone and smell detection returned to normal after 6 to 16 hours of treatment, auditory detection required at least 24 hours of treatment with $20 \mathrm{mg}$ of prednisolone before it returned to normal, and then the return was mainly in the low and middle frequency ranges. After treatment with carbohydrate-active steroids was discontinued, hearing sensitivity was markedly increased after 2 to 5 days, a phenomenon similar to that observed previously for both taste and smell $(1,2)$.

As with taste and smell, the ability to return auditory thresholds to normal is not limited to a single carbohydrate-active steroid, for thresholds have returned to normal after treatment with a variety of carbohydrate-active steroids. Furthermore, excessive amounts of steroid need not be administered to return auditory sensitivity to normal. At the time of admission the patients with ACI were on maintenance doses of carbohydrateactive steroids with or without sodium-potassiumactive steroids. At that time, auditory thresholds were similar to those measured after treatment with prednisolone, $20 \mathrm{mg}$ per day, for as long as 7 days.

Thresholds observed for the normal subjects in the present study are within the limits observed by many investigators, some of whom used techniques different from that used here (5-8). The average frequency range observed was from 50.5 to 15,500 cps. However, other investigators using other types of equipment have shown that normal subjects can respond to an average frequency range of 20 to $20,000 \mathrm{cps}(12,13)$. This difference in observed frequency response can be explained by the limitations of energy output of the earphones used in the present study. With these earphones, the frequency response of the patients with untreated ACI was significantly expanded over that of the normal subjects. This indicates that less sound energy was required by the patients with ACI to hear a given frequency than by the normal subjects; the earphone limitation imposed upon the normal subjects was not imposed upon the patients until higher or lower frequencies were reached.

Investigators have also noted that thresholds for higher frequencies decrease markedly as subjects become older (9-11). In the present study, there are significant differences between the auditory thresholds of the younger and older subjects (Table I). The upper limit of frequency response is markedly lower in the older subjects compared to the younger ones. However, auditory thresholds in the older patients, off treatment, for tones 500 to $2,000 \mathrm{cps}$ were significantly lower than even those of the younger normal subjects. The frequency response ranges for the older patients off treatment or on DOCA extended above those found after treatment with prednisolone or those of the older normal subjects by as much as 1,500 cps. This phenomenon was also observed in the younger patients. Thus, in spite of those changes that occur in the auditory sysem with age, e.g., presbycusis, the system in the older subjects is still responsive to the removal of carbohydrateactive steroid in the same qualitative, if not quantitative, manner as that of the younger patients.

Since hearing thresholds in the patients studied are significantly lower than normal, it might be reasonable to assume that they would be more aware of sounds around them than normal subjects. However, this is not the case, for none of them reported any excessive awareness of noise or gross discomfort with normal speech in the usual social setting. When the experimenter's voice was transmitted through the earphones at intensity levels only slightly above threshold to each patient in the sound chamber, the patients complained that the voice was rasping and uncomfortable. Speech patterns in all patients were grossly normal.

The mechanism by which this increase in sensitivity to sound occurs or its locus of action in the nervous system is not known. However, it is clear that this is not a phenomenon confined to the auditory system, since similar increases in sensitivity occur for the senses of taste and smell. Similarly, all three sensory modalities respond in the same general fashion to replacement with carbohydrate-active steroids. There may be a generalized increase in sensitivity to all sensory stimuli in untreated patients with ACI. Thus, 
steroid hormones may well play a significant role in maintaining the level of responsiveness of the sensory system to incoming stimuli. The manner in which this increase of sensitivity occurs and the behavioral and biochemical manifestations of these changes are presently being investigated (14-16).

\section{References}

1. Henkin, R. I., J. R. Gill, Jr., and F. C. Bartter. Studies on taste thresholds in normal man and in patients with adrenal cortical insufficiency: the role of adrenal cortical steroids and of serum sodium concentration. J. clin. Invest. 1963, 42, 727.

2. Henkin, R. I., and F. C. Bartter. Studies on olfactory thresholds in normal man and in patients with adrenal cortical insufficiency: the role of adrenal cortical steroids and of serum sodium concentration. J. clin. Invest. 1966, 45, 1631.

3. Stein, L., and S. Zerlin. Effect of circumaural earphones and earphone cushions on auditory threshold. J. acoust. Soc. Amer. 1963, 35, 1744.

4. Tillman, T. W., and K. D. Gish. Comments on the effect of circumaural earphones on auditory threshold. J. acoust. Soc. Amer. 1964, 36, 969.

5. Davis, H., and F. W. Kranz. The international standard reference zero for pure-tone audiometers and its relation to the evaluation of impairment of hearing. J. Speech Res. 1964, 7, 7.

6. Sivian, L. J., and S. D. White. On minimum audible sound fields. J. acoust. Soc. Amer. 1933, 4, 288.
7. VonBekesy, G., cited in H. Fletcher, Speech and Hearing in Communication. New York, Van Nostrand, 1953, p. 132.

8. Steinberg, J. C., H. C. Montgomery, and M. B. Gardner. Results of the World's Fair hearing tests. J. acoust. Soc. Amer. 1940, 12, 291.

9. Glorig, A., and H. Davis. Age, noise and hearing loss. Ann. Otol. (St. Louis) 1961, 70, 556.

10. Nixon, J. C., A. Glorig, and W. S. High. Changes in air and bone conduction thresholds as a function of age. J. Laryng. 1962, 76, 288.

11. Morgan, C. T. Physiological Psychology, 2nd ed. New York, McGraw-Hill, 1950.

12. Robinson, D. W., and R. S. Dadson. Threshold of hearing and equal-loudness relations for pure tones, and the loudness function. J. acoust. Soc. Amer. 1957, 29, 1284.

13. Davis, H., and S. R. Silverman. Hearing and Deafness. New York, Holt, Rinehart, and Winston, 1964, p. 44.

14. Henkin, R. I., R. L. Daly, and G. A. Ojemann. On the action of steroid hormones on the central nervous system in man (abstract). J. clin. Invest. $1966,45,1021$.

15. Henkin, R. I., and F. C. Bartter. The presence of corticosterone and cortisol in the central and peripheral nervous system of the cat. Program of the Endocrine Society 48th Meeting, Chicago, Ill., 1966 , p. 37.

16. Ojemann, G. A., and R. I. Henkin. Steroid dependent changes in human visual evoked potentials. Life Sci. In press. 